\title{
Correction to: The impact of nasal aspiration with an automatic device on upper and lower respiratory symptoms in wheezing children: a pilot case-control study
}

Antonio Pizzulli ${ }^{1}$, Serena Perna ${ }^{2}$, Anja Bennewiz ${ }^{3}$, Holger Roeblitz ${ }^{4}$, Salvatore Tripodi ${ }^{5}$, Jakob Florack², Petra Wagner ${ }^{2}$, Stephanie Hofmaier ${ }^{2}$ and Paolo Maria Matricardi ${ }^{2^{*}}$

\section{Correction}

The original article [1] contained a typesetting error in Table 5; this has now been corrected.

\section{Author details}

'Practice for Pediatric Allergy and Pneumology, Berlin, Germany.

${ }^{2}$ Department of Pediatric Pulmonology, Immunology and Intensive Care Medicine, Charité - University Medicine Berlin, Augustenburger Platz, 1, 13353 Berlin, Germany. ${ }^{3}$ Practice for General Pediatrics and Pediatric Cardiology, Berlin, Germany. ${ }^{4}$ Practice for General Pediatrics and Pediatric Allergy, Berlin,

Germany. ${ }^{5}$ Pediatric Department and Pediatric Allergology Unit, Sandro

Pertini Hospital, Rome, Italy.

Received: 25 June 2018 Accepted: 25 June 2018

Published online: 10 July 2018

\section{Reference}

1. Pizzulli A, et al. The impact of nasal aspiration with an automatic device on upper and lower respiratory symptoms in wheezing children: a pilot casecontrol study. Ital J Pediatr. 2018;44:68.

\footnotetext{
* Correspondence: paolo.matricardi@charite.de

${ }^{2}$ Department of Pediatric Pulmonology, Immunology and Intensive Care

Medicine, Charité - University Medicine Berlin, Augustenburger Platz, 1, 13353

Berlin, Germany
} 